\title{
MÉMOIRE SUR LES COURBES DU TROISIÈME ORDRE.
}

[From the Journal de Mathématiques Pures et Appliquées (Liouville), tome Ix. (1844),

pp. 285-293.]

Considérons d'abord la surface du troisième ordre qui passe par les six arêtes d'un tétraèdre quelconque. Cette surface sera touchée selon chaque arête par un seul plan; je dis que: "les plans tangents selon les arêtes opposées se rencontrent en trois droites qui sont dans le même plan, et chacune de ces droites est située entièrement sur la surface."

En effet, si nous représentons par $P=0, Q=0, R=0, S=0$, les équations des quatre plans du tétraèdre, et par $\alpha, 6, \gamma, \delta$ des constantes arbitraires, l'équation de la surface ne peut avoir que la forme

$$
\alpha Q R S+6 P R S+\gamma P Q S+\delta P Q R=0 .
$$

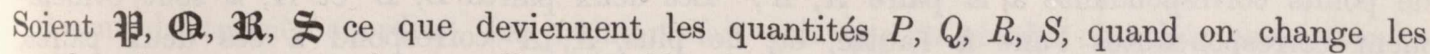
coordonnées $x, y, z$ en de nouvelles variables $\xi, \eta, \zeta$; l'équation du plan tangent se réduit facilement à la forme

$$
\begin{gathered}
(\text { 归 }-P)(6 R S+\gamma Q S+\delta Q R)+(\mathbb{Q}-Q)(\alpha R S+\gamma P S+\delta P R) \\
+(\mathfrak{R}-R)(\alpha Q S+6 P S+\delta P Q)+(\mathcal{D}-S)(\alpha Q R+6 P R+\gamma P Q)=0 .
\end{gathered}
$$

Soient $P=0, Q=0$; cette équation devient

$$
6 \text { 解 }+\alpha \text { 作 }=0 ；
$$

et de même, si $R=0, S=0$, l'équation devient

$$
\delta \mathfrak{R}+\gamma \dot{\Phi}=0 .
$$

De ces équations on déduit les deux suivantes:

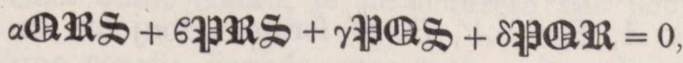

$$
\begin{aligned}
& \frac{1}{\alpha} \text { 非 }+\frac{1}{6} \mathbb{Q}+\frac{1}{\gamma} \mathfrak{R}+\frac{1}{\delta} \delta=0 \text {. }
\end{aligned}
$$


On conclut de la première, que la droite d'intersection des deux plans tangents est située sur la surface; et de la seconde, que cette droite est dans un même plan, quelles que soient les deux arêtes opposées par lesquelles on a mené les deux plans tangents. Ainsi le théorème est démontré.

En considérant une section quelconque de cette surface, ou même en supposant que $P, Q, R, S$ ne contiennent que deux variables $x, y$, nous avons ce théorème:

"Etant donnée une courbe du troisième ordre qui passe par les six points d'intersection de quatre droites, les tangentes à la courbe en ces six points se rencontrent deux à deux en trois points qui sont les points d'intersection de la courbe par une droite. Les tangentes qui doivent être prises ensemble sont les tangentes en deux points $A$, $A^{\prime}$, tels que $A$ est l'intersection de deux des quatre lignes données, et $A^{\prime}$ l'intersection des deux autres lignes, points que l'on peut nommer opposés.

"De même, si une courbe du troisième ordre passe par cinq de ces points, de telle manière que l'intersection des tangentes à la courbe en deux points opposés soit située sur la courbe, la courbe passe par le sixième point, et par les points d'intersection des tangentes menées par les deux autres paires de points opposés."

A présent, posons une courbe quelconque du troisième ordre et deux points $A, A^{\prime}$ sur la courbe, tels que les tangentes en ces deux points se rencontrent sur la courbe (cela suppose que la courbe est de la sixième ou quatrième classe, et non pas de la troisième). Un autre point $B$ sur la courbe étant pris à volonté, les droites $A B, A^{\prime} B$ rencontrent la courbe en $H$ et $h$; et les droites $A h, A^{\prime} H$ se rencontrent en un point $B^{\prime}$ situé sur la courbe. Les tangentes en $B, B^{\prime}$, et de même les tangentes en $H, h$, se rencontrent sur la courbe en deux points qui sont en ligne droite avec le point d'intersection des tangentes en $A$ et $A^{\prime}$.

On peut dire que les deux points $B, B^{\prime}$, ou les deux points $H, h$, sont une paire de points correspondante à la paire $A, A^{\prime}$. Les deux paires $B, B^{\prime}$ et $H, h$ sont évidemment correspondantes l'une à l'autre, et, de plus, $A, A^{\prime}$ correspond à ces deux paires, de la même manière que $H, h$ correspond à $A, A^{\prime}$, et $B, B^{\prime}$; de sorte que l'on peut dire que les trois paires $A, A^{\prime} ; B, B^{\prime} ; H, h$ sont supplémentaires l'une aux deux autres.

Soit $C, C^{\prime}$ une autre paire de points correspondante à $A, A^{\prime}$; je dis que $B, B^{\prime}$ et $C, C^{\prime}$ sont des paires correspondantes l'une à. l'autre; et, de plus, si d'un point $P$ quelconque de la courbe, l'on mène des lignes aux points $A, A^{\prime}, B, B^{\prime}, C, C^{\prime}$, ces lignes forment un faisceau en involution.

En effet, considérons six points quelconques $A, A^{\prime}, B, B^{\prime}, C, C^{\prime}$ dans le même plan, et représentons par $f, g, h$ les points d'intersection de $B C^{\prime}$ et $B^{\prime} C, C A^{\prime}$ et $C^{\prime} A, A B^{\prime}$ et $A^{\prime} B$, et par $F, G, H$ les points d'intersection de $C B$ et $C^{\prime} B^{\prime}, C A$ et $C^{\prime} A^{\prime}, A B$ et $A^{\prime} B^{\prime}$. Nous allons faire voir que le lieu d'un point $P$ qui se meut de telle manière que les lignes menées aux points $A, B, C, A^{\prime}, B^{\prime}, C^{\prime}$ forment toujours un faisceau en involution, est une courbe du troisième ordre qui passe par ces six points, et aussi par les points $f, g, h, F, G, H$. 
Soient $\alpha, \alpha^{\prime}, 6,6^{\prime}, \gamma, \gamma^{\prime}$ les tangentes trigonométriques des inclinaisons des lignes $P A, P A^{\prime}, P B, P B^{\prime}, P C, P C^{\prime}$, sur une ligne fixe quelconque que l'on peut prendre pour l'axe des $x$. Pour que ces lignes forment un faisceau en involution, nous devrions avoir la relation

$$
\alpha \alpha^{\prime}\left(\mathfrak{b}+\mathfrak{b}^{\prime}-\gamma-\gamma^{\prime}\right)+\mathscr{b} \mathscr{b}^{\prime}\left(\gamma+\gamma^{\prime}-\alpha-\alpha^{\prime}\right)+\gamma \gamma^{\prime}\left(\alpha+\alpha^{\prime}-6-b^{\prime}\right)=0,
$$

ou, ce qui revient à la même chose, l'une quelconque des quatre équations

$$
\begin{aligned}
& \left(\alpha-b^{\prime}\right)\left(6-\gamma^{\prime}\right)\left(\gamma-\alpha^{\prime}\right)+\left(\alpha^{\prime}-6\right)\left(b^{\prime}-\gamma\right)\left(\gamma^{\prime}-\alpha\right)=0, \\
& \left(\alpha^{\prime}-6^{\prime}\right)\left(6-\gamma^{\prime}\right)(\gamma-\alpha)+(\alpha-6)\left(6^{\prime}-\gamma\right)\left(\gamma^{\prime}-\alpha^{\prime}\right)=0, \\
& (\alpha-6)\left(6^{\prime}-\gamma^{\prime}\right)\left(\gamma-\alpha^{\prime}\right)+\left(\alpha^{\prime}-6^{\prime}\right)(6-\gamma)\left(\gamma^{\prime}-\alpha\right)=0, \\
& \left(\alpha^{\prime}-6\right)\left(6^{\prime}-\gamma^{\prime}\right)(\gamma-\alpha)+\left(\alpha-6^{\prime}\right)(6-\gamma)\left(\gamma^{\prime}-\alpha^{\prime}\right)=0 .
\end{aligned}
$$

Soient $x_{P}, y_{P}, x_{A}, y_{A}$, etc., les coordonnées de $P, A$, etc.

$$
\begin{aligned}
\alpha & =\frac{y_{P}-y_{A}}{x_{P}-y_{A}} \\
\alpha-G^{\prime} & =\frac{y_{P}-y_{A}}{x_{P}-x_{A}}-\frac{y_{P}-y_{B^{\prime}}}{x_{P}-x_{B^{\prime}}}=-\frac{1}{\left(x_{P}-x_{A}\right)\left(x_{P}-x_{B^{\prime}}\right)}\left(P A B^{\prime}\right),
\end{aligned}
$$

en représentant par $\left(P A B^{\prime}\right)$, etc., les quantités telles que

$$
x_{P}\left(y_{A}-y_{B^{\prime}}\right)+y_{P}\left(x_{A}-x_{B^{\prime}}\right)+x_{A} y_{B^{\prime}}-x_{B^{\prime}} y_{A^{*}},
$$

L'équation de la courbe peut donc se mettre sous l'une quelconque des quatre formes

$$
\begin{aligned}
& P A B^{\prime} \cdot P B C^{\prime} \cdot P C A^{\prime}+P A^{\prime} B \cdot P B^{\prime} C \cdot P C^{\prime} A=0, \\
& P A^{\prime} B^{\prime} \cdot P B C^{\prime} \cdot P C A+P A B \cdot P B^{\prime} C \cdot P C^{\prime} A^{\prime}=0, \\
& P A B . P B^{\prime} C^{\prime} \cdot P C A^{\prime}+P A^{\prime} B^{\prime} \cdot P B C \cdot P C^{\prime} A=0, \\
& P A^{\prime} B \cdot P B^{\prime} C^{\prime} \cdot P C A+P A B^{\prime} \cdot P B C \cdot P C^{\prime} A^{\prime}=0,
\end{aligned}
$$

qui sont du troisième ordre. La première fait voir que la courbe cherchée passe par les neuf points $A, B, C, A^{\prime}, B^{\prime}, C^{\prime}, f, g, h$. La troisième ou la quatrième fait voir qu'elle passe de plus par le point $F$; la quatrième ou la seconde, qu'elle passe par le point $G$; la seconde ou la troisième, qu'elle passe par le point $H$. Ainsi la courbe passe par les douze points $A, B, C, A^{\prime}, B^{\prime}, C^{\prime}, f, g, h, F, G, H$.

On peut remarquer qu'une courbe du troisième ordre qui passe par dix quelconques de ces points, ou même par neuf quelconques, pourvu que nous exceptions les combinaisons de $A, B, C, A^{\prime}, B^{\prime}, C^{\prime}$, avec $f, g, h$, ou $f, G, H$, ou $F, g, H$, ou $F, G, h$, ne peut être que la courbe que nous venons de trouver. On peut aussi remarquer en passant que si $A, A^{\prime}, B, B^{\prime}, C, C^{\prime}$ sont les points d'intersection de quatre äroites, l'équation cidevant trouvée est satisfaite identiquement, de sorte que la position du point $P$ est absolument arbitraire. Cela étant connu, je ne m'arrête pas pour le démontrer.

Revenons au cas d'une courbe donnée, avec trois paires de points $A, A^{\prime}, B, B^{\prime}, C, C^{\prime}$, comme auparavant, tels que $B, B^{\prime}$ et $C, C^{\prime}$ sont des paires correspondantes à $A, A^{\prime}$. Les 
points $A, B, C, A^{\prime}, B^{\prime}, C^{\prime}, G, g, H, h$ sont situés sur la courbe; ainsi $F, f$ sont aussi sur la courbe (c'est-à-dire que $B, B^{\prime}$ et $C, C^{\prime}$ sont des paires correspondantes), et les lignes menées par un point $P$ quelconque de la courbe et les points $A, B, C, A^{\prime}, B^{\prime}, C^{\prime}$ forment un faisceau en involution: théorème ci-devant énoncé.

Considérons une courbe du troisième ordre, de la quatrième classe (c'est-à-dire, telle que d'un point quelconque on ne peut lui mener que quatre tangentes). D'un point sur la courbe, indépendamment de la tangente en ce point, on ne peut mener que deux tangentes. Prenons les deux points $K, L$ sur la courbe, et menons les tangentes $K A$, $K A^{\prime}, L B, L B^{\prime}$ touchant la courbe en $A, A^{\prime}, B, B^{\prime}$. Il est clair qu'en ce cas $A, A^{\prime}$ et $B, B^{\prime}$ sont des paires correspondantes de points. Mais le cas général où la courbe est de la sixième classe est moins simple. Considérons, en effet, pour une telle courbe, les huit points $A_{1}, A_{2}, A_{3}, A_{4}$ et $B_{1}, B_{2}, B_{3}, B_{4}$ de contact des tangentes menées par les deux points $K$ et $L$. En choisissant $A, A^{\prime}$ et $B$ de quelque manière que ce soit, parmi les points $A_{1}, A_{2}, A_{3}, A_{4}$ et $B_{1}, B_{2}, B_{3}, B_{4}$ respectivement, le point $B^{\prime}$, qui doit entrer dans cette dernière série, ne peut pas être choisi à volonté, mais est parfaitement déterminé. En désignant convenablement les points $B$, on peut toujours supposer que les paires correspondantes söient $A_{1} A_{2}$ ou $A_{3} A_{4}$ avec $B_{1} B_{2}$ ou $B_{3} B_{4} ; A_{1} A_{3}$ ou $A_{2} A_{4}$ avec $B_{1} B_{3}$ ou $B_{2} B_{4} ; A_{1} A_{4}$ ou $A_{2} A_{3}$ avec $B_{1} B_{4}$ ou $B_{2} B_{3}$. Cela suppose que

$$
\begin{aligned}
& A_{1} B_{1}, \quad A_{2} B_{2}, \quad A_{3} B_{3}, \quad A_{4} B_{4} ; \quad A_{1} B_{2}, \quad A_{2} B_{1}, \quad A_{3} B_{4}, \quad A_{4} B_{3} ; \\
& A_{1} B_{3}, \quad A_{3} B_{1}, \quad A_{2} B_{4}, \quad A_{4} B_{2} ; \quad A_{1} B_{4}, \quad A_{4} B_{1}, \quad A_{2} B_{3}, \quad A_{3} B_{2} \text {, }
\end{aligned}
$$

se rencontrent, chaque système, dans le même point. Il faut expliquer avec plus d'evidence la corrélation de ces huit points.

Imaginons les six points $A, A^{\prime} ; B, B^{\prime} ; C, C^{\prime}$, tels que $A A^{\prime}, B B^{\prime}, C C^{\prime}$ se rencontrent dans le même point. Formons, comme auparavant, le système de points $f, g, h, F, G, H$. Les propriétés de ce système de douze points sont très-nombreuses. Non-seulement $F, G, H ; F, g, h ; f, G, h ; f, g, H$ sont en ligne droite; mais, en outre, les trois droites de chacun des onze systèmes que voici se rencontrent dans le même point:

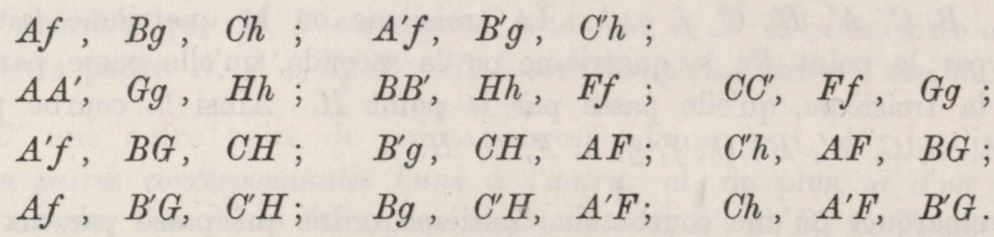

(Cela est connu, je crois; au reste, pour le démontrer, considérons un parallélipipède, tel que $g f, G F, A B, A^{\prime} B^{\prime}$ en soient quatre arêtes parallèles, les autres arêtes parallèles étant $g A, A^{\prime} G, f^{\prime} B, B^{\prime} F ; g A^{\prime}, A^{\prime} G, B f, B^{\prime} F$. Soient $H$ le point de rencontre, à l'infini, du premier système d'arêtes parallèles; $C^{\prime}$ et $C$ les points de rencontre, à l'infini, des arêtes des second et troisième systèmes respectivement; $h$ le point de rencontre des quatre diagonales du parallélipipède; faisons la perspective de ce système, désignant chaque point de la projection par la même lettre: l'on voit sans peine que la figure plane, ainsi obtenue, a les propriétés en question.) 
A présent, en examinant la figure, on voit qu'il est permis de prendre pour $A_{1}, A_{2}$, $A_{3}, A_{4}$ les points $A, A^{\prime}, F, f$, et pour $B_{1}, B_{2}, B_{3}, B_{4}$ les points $B, B^{\prime}, G, g$, pour que les systèmes $A_{1}, A_{2}, A_{3}, A_{4} ; B_{1}, B_{2}, B_{3}, B_{4}$ aient la corrélation ci-devant trouvée. Le système $C, C^{\prime}, H, h$ est supplémentaire à ces deux-ci.

Toutes les propriétés que je viens de trouver sont telles qu'il y a à chacune une propriété correspondante que l'on peut obtenir par la théorie des polaires réciproques. Nous avons ainsi des propriétés non moins intéressantes des courbes de la troisième classe et du sixième ou quatrième ordre.

En prenant pour la courbe du troisième ordre l'ensemble d'une section conique et d'une droite, pour que les tangentes en $A, A^{\prime}$ se rencontrent sur la courbe, il faut que $A, A^{\prime}$ soient situées sur la section conique de telle manière que $A A^{\prime}$ passe par le pôle de la droite à l'égard de la conique. Alors $B, B^{\prime} ; C, C^{\prime \prime}$ étant pris de la même manière, $B C^{\prime}, B^{\prime} C$ et $B C, B^{\prime} C^{\prime}$ se rencontrent sur la droite.

Les lignes tirées d'un point $P$ quelconque de la conique, ou de la droite à $A, B, C$; $A^{\prime}, B^{\prime}, C^{\prime}$, forment un faisceau en involution.

Le premier théorème et la première partie du second sont très-bien connus; je ne sais s'il en est de même de la dernière partie de ce théorème.

\section{ADDITION.}

La droite $\mathrm{PP}^{\prime}$, menée par deux points $\mathrm{P}, \mathrm{P}^{\prime}$ d'une courbe du troisième ordre, qui correspondent toujours d̀ une paire correspondante donnée de points $\mathrm{A}, \mathrm{A}^{\prime}$, est toujours tangente d̀ une certaine courbe de la troisième classe.

Pour démontrer ce théorème, imaginons une paire fixe $B, B^{\prime}$ de points qui corresponde à la paire donnée $A, A^{\prime}$. Soient $P=0, Q=0, R=0, S=0$ les équations des lignes qui joignent $A, A^{\prime}$ avec $B, B^{\prime}$; l'équation de la courbe donnée du troisième ordre peut se mettre sous la forme

$$
\frac{\alpha}{P}+\frac{\ell}{Q}+\frac{\gamma}{R}+\frac{\delta}{S}=0
$$

On peut toujours supposer, sans perte de généralité, que l'équation

$$
P+Q+R+S=0
$$

soit identiquement vraie, car chaque équation de la forme $P=0$ peut être censée contenir une constante arbitraire qui en multiplie tous les termes.

Donc, en faisant

$$
P+R=\theta, \quad P=\theta-R,
$$

on peut toujours supposer

$$
Q+S=-\theta, \quad Q=-\theta-S
$$


et l'équation de la courbe se transforme en

$$
\frac{\alpha}{\theta-R}-\frac{6}{\theta+S}+\frac{\gamma}{R}+\frac{\delta}{S}=0
$$

ce que l'on peut écrire aussi sous la forme

$$
\frac{\theta+(\lambda \mu+\lambda+\mu) S}{(\theta+\lambda R)(\theta+\mu R)}+\frac{-\theta+(\nu \rho+\nu+\rho) R}{(-\theta+\nu S)(-\theta+\rho S)}=0
$$

en déterminant convenablement les constantes $\lambda, \mu, \nu, \rho$. En effet, en réduisant, lés deux équations deviennent identiques au moyen des quatre conditions

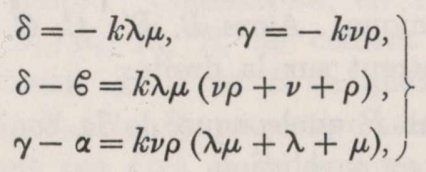

où $k$ est une quantité arbitraire, de manière que des quantités $\lambda, \mu, \nu, \rho$, il y en a une seule qui peut être prise à volonté.

De l'équation (4) l'on déduit tout de suite cette autre forme,

$$
\frac{1}{\mu-\lambda}\left[\frac{\mu(\lambda+1)}{\theta+\lambda R}-\frac{\lambda(\mu+1)}{\theta+\mu R}\right]+\frac{1}{\rho-\nu}\left[\frac{\rho(\nu+1)}{-\theta+\nu S}-\frac{\nu(\rho+1)}{-\theta+\rho S}\right]=0
$$

et de là nous voyons que les points donnés par les deux systèmes d'équations

$$
\left.\begin{array}{l}
(\theta+\lambda R=0, \quad-\theta+\nu S=0) \\
(\theta+\mu R=0, \quad-\theta+\rho S=0)
\end{array}\right\}
$$

correspondent toujours l'un à l'autre et aux points donnés par les deux systèmes

$$
\left.\begin{array}{ll}
(\theta=0, & R=0) \\
(\theta=0, & S=0)
\end{array}\right\}
$$

c'est-à-dire aux points $A, A^{\prime}$.

On trouve assez facilement pour l'équation de la droite menée par les points déterminés par les deux systèmes (7), points que l'on peut prendre pour $P$ et $P^{\prime}$,

$$
(\mu \nu-\rho \lambda) \theta+\lambda \mu(\nu-\rho) R+\nu \rho(\lambda-\mu) S=0 .
$$

ou

$$
C \theta+A R+B S=0
$$

en faisant

$$
\left.\begin{array}{l}
p C=\mu \nu-\rho \lambda \\
p A=\lambda \mu(\nu-\rho) \\
p B=\nu \rho(\lambda-\mu)
\end{array}\right\}
$$


Éliminons des équations (5) et (11) les cinq quantités $\lambda, \mu, \nu, \rho, p$. Pour opérer de la manière la plus élégante, formons d'abord les équations identiques

$$
\left.\begin{array}{c}
{[\mu \nu-\rho \lambda-\nu \rho(\lambda-\mu)](\nu-\rho)(\lambda \mu+\lambda+\mu)} \\
+[\mu \nu-\rho \lambda+\lambda \mu(\nu-\rho)](\lambda-\mu)(\nu \rho+\nu+\rho) \\
=(\nu \lambda-\mu \rho)[\lambda \mu(\nu-\rho)-\nu \rho(\lambda-\mu)+2(\mu \nu-\rho \lambda)], \\
\lambda \mu(\nu-\rho)^{2}-\nu \rho(\lambda-\mu)^{2}=(\mu \nu-\rho \lambda)(\lambda \nu-\mu \rho),
\end{array}\right\}
$$

ce qui donne

$$
\begin{array}{r}
A(C-B)(\gamma-\alpha)+B(C+A)(\delta-6) \\
=\frac{k}{p} \lambda \mu \nu \rho(\nu \lambda-\mu \rho)(2 C+A-B), \\
A^{2} \gamma-B^{2} \delta=-\frac{k}{p} \lambda \mu \nu \rho(\nu \lambda-\mu \rho) C
\end{array}
$$

et de là

$$
\left.\begin{array}{c}
C[A(C-B)(\gamma-a)+B(C+A)(\delta-6)] \\
+(2 C+A-B)\left(A^{2} \gamma-B^{2} \delta\right)
\end{array}\right\}=0
$$

ou, toute réduction faite,

$$
\left.\begin{array}{r}
A(A+C)(A+C-B \gamma)+B(C-B)(A+B-C \delta) \\
-A C(C-B \alpha)-B C(C+A 6)
\end{array}\right\}=0
$$

et puisque cette équation est du troisième ordre à l'égard des quantités $A, B, C$, il est évident que la ligne donnée par l'équation (10) est toujours tangente à une certaine courbe de la troisième classe. En supposant $\gamma=0, \delta=0$, ce qui réduit la courbe du troisième ordre aux lignes $R=0, S=0,(6-\alpha) \theta-6 R-\alpha S=0$, l'équation (15) se partage dans les deux équations

$$
C=0, \quad A(C-B) \alpha+B(C+A) b=0
$$

et la courbe de la troisième classe se réduit au point $(R=0, S=0)$ et à une conique. Cela est un théorème connu, car l'on sait bien que si $A, A^{\prime}$ sont des points quelconques sur deux droites données $\alpha$, $\alpha^{\prime}$, et $B, B^{\prime}$ deux autres points déterminés, sur ces droites, de manière que l'intersection des lignes $A B^{\prime}, A^{\prime} B$ soit toujours sur une ligne droite donnée, les deux lignes $\alpha, \alpha^{\prime}$ sont divisées homographiquement, $B, B^{\prime}$ étant deux points correspondants; et de plus, que la ligne qui unit les points correspondants de deux lignes divisées homographiquement, est toujours tangente à une certaine conique. Quant au point d'intersection des lignes $\alpha, \alpha^{\prime}$, que nous venons de trouver comme formant avec la conique une courbe de la troisième classe, on observera que, dans notre théorie, nonseulement les points des lignes $\alpha, \alpha^{\prime}$ se correspondent, mais que le point d'intersection des lignes $\alpha, \alpha^{\prime}$ correspond à tous les points de la troisième droite. La conique touche les deux droites $\alpha, \alpha^{\prime}$; cela n'a pas, je crois, d'analogie dans la théorie générale. 